\title{
Adsorção de Cd(II) por lama vermelha natural e com diferentes ativações
}

\section{Mariana S. G. da Silva* \\ Beatriz C. Pichinelli \\ Fabiano T. da Conceição \\ Rodrigo B. Moruzzi \\ Lauren N. M. Yabuki \\ Amauri A. Menegário \\ Maria Lúcia P. Antunes.}

Departamento de Planejamento Territorial e Geoprocessamento (DEPLAN)

IGCE - UNESP

Campus de Rio Claro (SP)

Rio Claro - SP

Avenida 24 A, 1515

CEP13506-900

Fone: (19) 3526-9358.

* Autora correspondente

marianascicia@hotmail.com

\begin{abstract}
RESUMO
Alguns elementos são considerados tóxicos mesmo em baixas concentrações, causando diversos problemas de saúde pública. O cádmio é amplamente utilizado em atividades industriais e pode causar contaminação do solo e da água. A lama vermelha foi estudada para uso como adsorvente na remoção de elementos, compostos de águas residuais e/ou de solo contaminado. No entanto, existem vários compostos e tratamentos que ainda não foram testados. Neste estudo, o potencial de adsorção Cd (II) para lama vermelha natural (LV) e lama vermelha ativada por tratamento térmico a $400^{\circ} \mathrm{C}(\mathrm{LVT})$ e tratamento químico com $\mathrm{HCl}$ a $0,05 \mathrm{~mol} \mathrm{~L}^{-1}(\mathrm{LVQ} 1)$ e com $\mathrm{Ca}\left(\mathrm{NO}_{3}\right)_{2} 0,1$ mol L ${ }^{-1}$ (LVQ2) foi avaliado. A LV e a LVT apresentaram maior percentual de adsorção de Cd (II) em solução aquosa. A isoterma de Freundlich foi mais apropriada para descrever o fenômeno da remoção de $\mathrm{Cd}$ (II), a lama vermelha ativada termicamente teve a maior capacidade de adsorção, 1,04 mmol de $\mathrm{g}^{-1}$ ou $107,52 \mathrm{mg} \mathrm{de}^{-1}$. Esses resultados indicam que a LVT tem potencial para uso em aplicações que tratam efluentes e/ou solo contaminado da atividade industrial.
\end{abstract}

Palavras-chave: Cd (II), lama vermelha, adsorção, cinética, termodinâmica.

\section{ABSTRACT}

Some elements are considered toxic even in low concentrations, causing various public health problems. Cadmium is widely used in industrial activities and can cause contamination of soil and water. The red mud was studied for use as adsorbent in the removal of elements, composed of wastewater and/or contaminated soil. However, there are several compounds and treatments that have not yet been tested. In this study, the potential of $\mathrm{Cd}$ (II) adsorption for natural red mud (LV) and red mud activated by thermal treatment at $400^{\circ} \mathrm{C}(\mathrm{LVT})$ and chemical treatment with $0.05 \mathrm{~mol} \mathrm{~L}^{-1} \mathrm{HCl}$ (LVQ1) and with $\mathrm{Ca}\left(\mathrm{NO}_{3}\right)_{2} 0.1 \mathrm{~mol} \mathrm{~L}^{-1}$ (LVQ2) was evaluated. The $\mathrm{LV}$ and LVT presented higher percentage of Cd (II) adsorption in aqueous solution. The Freundlich isotherm was more appropriate to describe the phenomenon of Cd (II) removal, the thermally activated red mud had the highest adsorption capacity, $1.04 \mathrm{mmol} \mathrm{g}^{-1}$ or $107.52 \mathrm{mg} \mathrm{g}^{-1}$. These results indicate that LVT has potential for use in applications that treat effluents and / or contaminated soil from industrial activity.

Keywords: Cd (II), red mud, adsorption, kinetics, thermodynamics.

\section{INTRODUÇÃO}

Diversas indústrias são responsáveis pelo descarte de águas residuárias com constituintes que podem ocasionar efeitos adversos ao meio ambiente, se lançados nos recursos hídricos ou em sistemas públicos de esgotos sanitários sem os devidos tratamentos estabelecidos em normas e legislações específicas. Dentre os constituintes desses efluentes industriais, destacam-se os metais pesados, poluentes não compatíveis com tratamentos biológicos, que sofrem o efeito da amplificação biológica, além de causarem efeitos carcinogênicos e até a morte. O cádmio $(\mathrm{Cd})$ é 
considerado um dos metais mais tóxicos, sendo matéria prima em diversas atividades industriais, tais como galvanoplastia e produção de ligas, baterias, pigmentos, componentes eletrônicos e nucleares.

No âmbito do tratamento de despejos industriais e esgoto sanitário, vários métodos têm sido utilizados para remoção de metais, dentre eles: precipitação química, troca iônica, osmose reversa e adsorção. Dentre esses métodos destaca-se a adsorção como sendo uma técnica eficiente, de fácil manipulação, com uma grande variedade de possíveis adsorventes e bom custo-benefício (Nadaroglu et al. 2010). Atualmente, os adsorventes mais empregados são aqueles à base de carvão ativado, porém, eles são relativamente caros, com pouca seletividade e baixa eficiência de remoção de contaminantes inorgânicos (Smiljanic et al., 2010). Desta forma, há uma crescente preocupação em desenvolverem-se novos adsorventes de metais pesados utilizando-se materiais disponíveis, como os resíduos industriais. Estudos realizados mostram o uso de cinzas volantes, lamas de altoforno, lodos, resíduos de fábricas de chá, polpa de beterraba, entre outros, como adsorventes de baixo-custo de metais pesados em águas residuárias, além da lama vermelha, ou red mud, um dos mais promissores adsorventes da atualidade (Ahmaruzzaman, 2011).

A lama vermelha é um resíduo insolúvel gerado no processo de obtenção do alumínio da bauxita, por meio do processo Bayer, o qual é disposto em locais adequados denominados lagoas de disposição. A lama vermelha possui elevado teor alcalino e é composta por finas partículas de óxidos e hidróxidos de sílica, alumínio, ferro, cálcio e titânio, podendo também conter elementos traços de óxidos de outros metais (Hind et al. 1999). A quantidade de lama vermelha gerada durante o beneficiamento pode chegar ao dobro da quantidade de alumina produzida, o que demanda uma grande área de disposição, altos custos e riscos ambientais para a indústria do alumínio (Hind et al. 1999). A lama vermelha não é classificada como um rejeito perigoso, mas sua disposição inadequada pode acarretar em problemas como contaminação de águas superficiais e subterrâneas por $\mathrm{NaOH}, \mathrm{Fe}, \mathrm{Al}$ ou outro agente químico e danos à flora e fauna da região de entorno das lagoas de disposição (Hind et al. 1999; Silva Filho et al. 2007).

Estudos apontam que a lama vermelha apresenta propriedades de adsorção, principalmente quando ativada térmica ou quimicamente, constituindo-se em um adsorvente de baixo custo de obtenção, que pode ser empregado no tratamento de águas de abastecimento e residuárias, adsorvendo metais e íons metálicos tóxicos, ânions inorgânicos e orgânicos, e bactérias (Rai et al., 2012). Trabalhos recentes na literatura apresentam seu potencial de adsorção para metais, arsênio (As), fluoreto e corantes (Akin et al., 2012; Conceição et al., 2016; Costa et al., 2009; Fu et al., 2010; Geyikçi et al., 2012.; Grudić et al., 2013; Jesus, 2011; Liang et al., 2014; Nadaroglu et al., 2010; Pichinelli et al., 2017; Pulford et al., 2012; Sahu, Patel \& Ray, 2011; Ratnamala et al. 2012; Smiciklas et al., 2014; Smiljanic et al., 2010; Souza et al., 2013; Souza et al. 2013).

Além disso, estudos visando a neutralização da lama vermelha também veem sendo realizados, tais como lavagem simples com água, fervura com ácido, ativação com ácido e com $\mathrm{CO}_{2}$ (Apak et al. 1998a; Apak et al., 1998b; Cengeloglu et al., 2006; Grudić et al., 2013; Liang et al., 2014; Nadaroglu, Kalkan \& Demir, 2010; Sahu et al., 2011; Santona et al., 2006; Smiciklas et al., 2014;). Ainda há outras formas de ativação química como a utilização de água marinha, térmica, com temperaturas variando de 200 a $900^{\circ} \mathrm{C}$ e com peróxido de hidrogênio (Apak et al., 1998a; Erdem et al., 2004; Grudić et al., 2013; Gupta \& Sharma, 2002; Palmer et al., 2010; Smiljanic et al., 2010; Souza et al., 2013; Souza et al., 2013).

Assim, o objetivo deste estudo foi caracterizar a lama vermelha $(\mathrm{LV})$ e a lama vermelha ativada por tratamento térmico a $400^{\circ} \mathrm{C}$ (LVT) e químico com ácido clorídrico $(\mathrm{HCl})$ $0.05 \mathrm{~mol} \mathrm{~L}^{-1}$ (LVQ1) e nitrato de cálcio $\left[\mathrm{Ca}\left(\mathrm{NO}_{3}\right)_{2}\right] 0.1 \mathrm{~mol} \mathrm{~L}^{-1}$ (LVQ2), além de avaliar o potencial de adsorção de Cd(II) de cada material estudado usando isotermas de adsorção de Langmuir e Freundlich. Ainda, foram realizados testes para verificar a influência do $\mathrm{pH}$, da cinética de adsorção e da termodinâmica, permitindo identificar se estes materiais pode ser considerados uma alternativa para remediação de áreas contaminadas e tratamento de efluentes industriais. 


\section{MATERIAIS E MÉTODOS}

\subsection{ATIVAÇÃO QUÍMICA DA LAMA VERMELHA}

A lama vermelha utilizada neste estudo foi coletada em uma indústria produtora de alumínio localizada no estado de São Paulo. As amostras de lama vermelha natural (LV) a serem ativadas foram destorroadas em cadinhos de porcelana e passadas em peneira com aberturas de $150 \mu \mathrm{m}$. Para a ativação com ácido clorídrico (LVQ1), as amostras da lama vermelha natural foram colocadas em béqueres, misturadas ao $\mathrm{HCl} 0,05$ $\mathrm{N}$ na proporção de 1:25 (grama de lama vermelha por $\mathrm{mL}$ de $\mathrm{HCl} 0,05 \mathrm{~N}$ ). A mistura foi colocada na mesa agitadora por 2 horas. Em seguida, permaneceram em repouso para

decantação da lama ativada. Então, o sobrenadante foi retirado e à lama vermelha restante foi acrescentada água destilada, repetindo-se o processo por mais uma vez. A lama restante foi seca durante 12 horas a $60^{\circ} \mathrm{C}$ em uma estufa. Outro tratamento da lama vermelha natural foi feito com $\mathrm{Ca}\left(\mathrm{NO}_{3}\right)_{2} \quad 0,1 \mathrm{~N}$ (LVQ2), e seguiu os mesmos procedimentos da ativação com $\mathrm{HCl} 0,05 \mathrm{~N}$. No tratamento térmico da lama vermelha natural (LVT), as amostras foram colocadas em cadinhos de porcelana e levadas à mufla à temperatura de $400{ }^{\circ} \mathrm{C}$, onde permaneceram durante duas horas.

\subsection{CARACTERIZAÇÃO DAS DIFERENTES LAMAS VERMELHAS}

Todos os ensaios de caracterização foram realizados para a LV, LVT, LVQ1 e LVQ2. Os valores de $\mathrm{pH}$ e condutividade elétrica $(\mathrm{CE})$ foram determinados na proporção 1:25 (grama de lama vermelha por $\mathrm{mL}$ de água destilada e deionizada), de acordo com a metodologia de Embrapa (EMBRAPA, 1997). A determinação da capacidade de troca catiônica (CTC), em mmol(+) $\mathrm{kg}^{-1}$, também foi realizada de acordo com o procedimento de Embrapa (EMBRAPA, 1997). A área específica superficial (ASE) foi obtida a partir de curvas de adsorção de nitrogênio. Para isso, as amostras foram degaseificadas a $200^{\circ} \mathrm{C}$, durante um período de 8 horas, e as isotermas de adsorção de nitrogênio, à temperatura de $196^{\circ} \mathrm{C}$ e faixa de vapor de 0,01 a 0,99 , foi realizada utilizando-se o equipamento Micromeritics - Asap Tristar 3000. Os valores das áreas superficiais específicas e do tamanho dos grãos foram determinados por meio do

\subsection{ESTUDO DA ADSORÇÃO DE CÁDMIO}

Para a análise da influência do $\mathrm{pH}$ na adsorção de Cd(II) pela lama vermelha e suas variações ativadas, foram realizados testes de adsorção em cinco diferentes valores de $\mathrm{pH}: 2,4$, 7, 10 e 12. Para isso, misturou-se $1 \mathrm{~g}( \pm 0,01 \mathrm{~g})$ de amostra à solução do metal na concentração de $1 \mathrm{mmol} 25 \mathrm{~mL}^{-1}$. As soluções de Cd(II) foram obtidas a partir de nitrato de cádmio tetrahidratado, $\mathrm{Cd}\left(\mathrm{NO}_{3}\right)_{2} \cdot 4 \mathrm{H}_{2} \mathrm{O}$. As amostras modelo matemático de Brunauer, Emmett e Teller (BET) (Brunauer et al., 1938).

A composição química das amostras de lama vermelha e suas variações ativadas foram obtidas por Espectrometria de Fluorescência de Raios X (FRX) no equipamento PHILIPS PW 2400, sendo anaisados os sehuintes parâmetros: $\mathrm{SiO}_{2}$, $\mathrm{Al}_{2} \mathrm{O}_{3}, \mathrm{Fe}_{2} \mathrm{O}_{3}, \mathrm{TiO}_{2}, \mathrm{~K}_{2} \mathrm{O}, \mathrm{Na}_{2} \mathrm{O}, \mathrm{MgO}, \mathrm{MnO}$, $\mathrm{CaO}, \mathrm{P}_{2} \mathrm{O}_{5}$ e LOI (perda ao fogo a $1000^{\circ} \mathrm{C}$ ). As análises mineralógicas por difração de raios $\mathrm{X}$ (DRX) foram realizadas em um difratômetro Siemens D5000, medidas com radiação de $\mathrm{Cu}$ $(\mathrm{WL}=1,542 \AA)$ e filtro de Ni. A velocidade do goniômetro foi definida com 3 graus por minuto e tempo de exposição de $1 \mathrm{~s}$ por cada passo de $0,05^{\circ}$. Além disso, foram obtidas micrografias por meio de elétrons secundários (SEI) utilizando-se o Microscópio Eletrônico de Varredura (MEV) JEOL JSM-6010LA, acoplando com um sistema de espectrometria de dispersão de energia (EDS).

foram agitadas durante 5 horas a $145 \mathrm{rpm}$ em mesa agitadora e a cada 15 minutos foi feito o controle do $\mathrm{pH}$, com soluções diluídas de ácido clorídrico e hidróxido de sódio.

Para a elaboração das isotermas, foram feitos testes de adsorção variando-se as concentrações iniciais de $\mathrm{Cd}(\mathrm{II})$ para uma mesma massa de lama vermelha. $\mathrm{O}$ experimento foi realizado com soluções aquosas preparadas com oito diferentes 
concentrações, a partir do sal do metal, variando de $0,5 \mathrm{mmol} 25 \mathrm{~mL}^{-1}$ a $4,0 \mathrm{mmol} 25 \mathrm{~mL}^{-1}$, em pH 5,0-5,5.25 Em relação aos estudos cinéticos, foi analisada a variação da concentração remanescente de $\mathrm{Cd}(\mathrm{II})$ no decorrer do tempo, em $\mathrm{pH}$ 5,0-5,5. Para isso, foram preparadas misturas contendo $1 \mathrm{~g}( \pm 0,01 \mathrm{~g})$ da amostra em soluções aquosas de $1 \mathrm{mmol} 25 \mathrm{~mL}^{-1}$ de $\mathrm{Cd}(\mathrm{II})$. As amostras também foram colocadas na mesa agitadora a $145 \mathrm{rpm}$ e retiradas em tempos distintos $(15,30,60,120,420,660$ e 1440 minutos). A influência da temperatura na adsorção foi estudada por testes de adsorção em temperaturas de $303 \mathrm{~K}\left(30^{\circ} \mathrm{C}\right), 313 \mathrm{~K}\left(40^{\circ} \mathrm{C}\right)$ e $323 \mathrm{~K}\left(50^{\circ} \mathrm{C}\right)$, usando $1 \mathrm{~g}( \pm 0,01 \mathrm{~g})$ de amostra em solução aquosa de $1 \mathrm{mmol} 25 \mathrm{~mL}^{-1}$ de Cd(II), em $\mathrm{pH} 5,0-5,5$, com as amostras agitadas a 145 rpm, por 7 horas.

Após cada experimento, as amostras foram centrifugadas por 25 minutos a $3000 \mathrm{rpm}$, e as alíquotas analisadas em relação à concentração de $\mathrm{Cd}(\mathrm{II})$ remanescente no sobrenadante. A determinação da concentração do $\mathrm{Cd}$ (II) foi realizada por espectrometria de emissão ótica com plasma indutivamente acoplado (ICP OES), usando o equipamento iCAP 6000 SERIES machine Thermo Scientific, com limite de detecção de $0,002 \mathrm{mg} \mathrm{L}^{-1}$. Todos os experimentos foram realizados em triplicatas.

A quantidade de metal adsorvida por unidade de adsorvente, $\mathrm{q}_{\mathrm{e}}$, em mmol $\mathrm{g}^{-1}$, foi calculada por meio da Equação 1. Para os cálculos de porcentagem de adsorção (\% Ads) foi utilizada a Equação 2.

$$
\begin{aligned}
& q_{e}=\frac{C_{0}-C_{e}}{m} V \\
& \% \text { Ads }=\frac{C_{0}-C_{e}}{C_{0}} 100
\end{aligned}
$$

Onde: $\mathrm{C}_{0}=$ concentração inicial do metal (mmol $\left.25 \mathrm{~mL}^{-1}\right) ; \mathrm{C}_{\mathrm{e}}=$ concentração final do metal no equilíbrio (mmol $\left.25 \mathrm{~mL}^{-1}\right) ; \mathrm{m}=$ massa do adsorvente $(\mathrm{g}) ; \mathrm{V}=$ volume da solução $(\mathrm{mL})$.

\subsection{ANÁLISE DO AJUSTE MATEMÁTICO AOS DADOS EXPERIMENTAIS}

O ajuste dos modelos aos dados experimentais foi avaliado por meio do erro relativo médio (ERM). Os valores de ERM foram calculados pela Equação 3 (Behnamfard et al., 2014). Esses valores podem ser negativos ou positivos, indicando superestimação ou subestimação do modelo empregado, respectivamente, em relação aos dados experimentais. Quanto menor for $\mathrm{o}$ valor absoluto de ERM, mais precisa é a estimativa do valor de qe pelo modelo. O coeficiente de

\section{RESULTADOS E DISCUSSÃO}

\subsection{CARACTERIZAÇÃO DA LAMA VERMELHA}

Na Tabela 1 são apresentados os resultados obtidos durante a caracterização da lama vermelha e suas variações ativadas. A LV possui $\mathrm{pH}$ alcalino $(10,2)$ e EC de $3800 \mu \mathrm{S} \mathrm{cm}^{-1}$. Após as ativações, os valores de $\mathrm{pH}$ e EC decrescem para LVQ1, LVQ2 e LVT. Os valores de CTC foram praticamente idênticos, variando entre 108,5 (LVT) e 112,0 (LVQ1) mmol(+) $\mathrm{kg}^{-1}$. Os menores valores de ASE foram determinados determinação da reta $\left(\mathrm{R}^{2}\right)$ empregado na avaliação dos ajustes (Behnamfard et al., 2014).

$$
E R M=\frac{100}{N} \sum_{i=1}^{N}\left(\frac{q_{e \text { exp }}-q_{e c a l}}{q_{\text {e exp }}}\right) i
$$

Onde: $\mathrm{qe}_{\exp }=$ quantidade de adsorvato por unidade de adsorvente experimental $\left(\mathrm{mmol} \mathrm{g}^{-1}\right)$; $\mathrm{qe}_{\text {cal }}=$ quantidade de adsorvato por unidade de adsorvente calculada de acordo com o modelo $\left(\mathrm{mmol} \mathrm{g}{ }^{-1}\right) ; \mathrm{N}=$ número de medições realizadas.

para a LV (31,0 $\left.\mathrm{m}^{2} \mathrm{~g}^{-1}\right)$ e LVQ1 $\left(79,0 \mathrm{~m}^{2} \mathrm{~g}^{-1}\right)$, respectivamente. $\mathrm{O}$ tamanho dos poros variou entre 1,0 e 4,5 $\mu \mathrm{m}$, sendo as lamas vermelhas compostas por partículas com diferentes tamanhos, formas e texturas (Figura 1), uma vez que as lamas vermelhas são constituídas por materiais heterogêneos com minerais variando de $<1 \mu \mathrm{m}$ a $>10 \mu \mathrm{m}$ de diâmetro. A morfologia dos minerais não foi alterada após as ativações. 
Tabela 1 - Caracterização das diferentes lamas vermelhas usadas neste estudo.

\begin{tabular}{ccccc}
\hline Parâmetro & LV & LVQ1 & LVQ2 & LVT \\
\hline $\mathrm{pH}$ & 10,2 & 8,7 & 8,1 & 10,0 \\
$\mathrm{CE}\left(\mu \mathrm{S} \mathrm{cm}^{-1}\right)$ & 3800,0 & 270,0 & 138,0 & 1700,0 \\
$\mathrm{CTC}\left(\mathrm{mmol}_{(+)} \mathrm{kg}^{-1}\right)$ & 109,0 & 112,0 & 109,5 & 108,5 \\
$\mathrm{ASE}\left(\mathrm{m}^{-1} \mathrm{~g}^{-1}\right)$ & 31,0 & 79,0 & 40,0 & 56,0 \\
Tamanho dos poros $(\mu \mathrm{m})$ & $3,0 \mathrm{a} 4,0$ & $3,0 \mathrm{a} 4,0$ & $3,0 \mathrm{a} 4,0$ & 1,0 a 4,0 \\
\hline
\end{tabular}

A composição química da lama vermelha e suas variações ativadas podem ser visualizadas na Tabela 2. Os principais componentes são óxido de ferro, alumina e sílica, o que está de acordo com resultados encontrados na literatura para a lama sem tratamento (Smiljanic et al., 2010; Souza et al., 2013). Os resultados referentes à difração de raios-X (Figura 2) indicam que a $\mathrm{LV}$ é constituída por gibbsita $\left(\mathrm{Al}(\mathrm{OH})_{3}\right)$, caulinita $\left(\mathrm{Al}_{2} \mathrm{Si}_{2} \mathrm{O}_{5}(\mathrm{OH})_{4}\right)$, quartzo $\left(\mathrm{SiO}_{2}\right)$, goethita $(\mathrm{FeOOH})$, dickita $\left(\mathrm{Al}_{2} \mathrm{Si}_{2} \mathrm{O}_{5}(\mathrm{OH})_{4}\right)$, componente do grupo caulinita, hematita $\left(\mathrm{Fe}_{2} \mathrm{O}_{3}\right)$, sodalita $\left(\mathrm{Na}_{8} \mathrm{Al}_{6} \mathrm{Si}_{6} \mathrm{O}_{24} \mathrm{Cl}_{2}\right)$ e calcita $\left(\mathrm{CaCO}_{3}\right)$. A mesma mineralogia foi observada para as amostras LVQ1 e LVQ2, com exceção da presença da calcita na LVQ2, formada durante o processo de neutralização com $\mathrm{Ca}\left(\mathrm{NO}_{3}\right)_{2}$ (Pichinelli et al., 2017). Para a LVT, o tratamento termal foi responsável pela conversão de goethita para hematita a $234^{\circ} \mathrm{C}\left(\mathrm{FeO}(\mathrm{OH}) \rightarrow \mathrm{Fe}_{2} \mathrm{O}_{3}\right)$ e de gibbsita para alumina a $272^{\circ} \mathrm{C}\left(\mathrm{Al}(\mathrm{OH})_{3} \rightarrow\right.$ $\mathrm{Al}(\mathrm{OH})(\mathrm{S})+\mathrm{H}_{2} \mathrm{O} \rightarrow \mathrm{Al}_{2} \mathrm{O}_{3}+\mathrm{H}_{2} \mathrm{O}$ (Antunes et al., 2012). Com isso, a predominância de alumina na lama vermelha promove o aumento na ASE na LVT em relação à $L V$, visto que a alumina possui ASE maior em relação as formas originais de hidróxido de alumínio.

Tabela 2 - Composição química (\%) das diferentes lamas vermelhas usadas neste estudo.

\begin{tabular}{ccccc}
\hline Óxido & LV & LVQ1 & LVQ2 & LVT \\
\hline $\mathrm{SiO}_{2}$ & 16,06 & 14,85 & 16,56 & 16,29 \\
$\mathrm{TiO}_{2}$ & 11,20 & 8,72 & 7,39 & 8,66 \\
$\mathrm{Al}_{2} \mathrm{O}_{3}$ & 15,50 & 16,49 & 25,06 & 17,50 \\
$\mathrm{Fe}_{2} \mathrm{O}_{3}$ & 38,56 & 35,06 & 39,57 & 35,77 \\
$\mathrm{MnO}$ & 0,28 & 0,27 & 0,40 & 0,24 \\
$\mathrm{MgO}$ & 0,14 & 0,12 & 0,11 & 0,10 \\
$\mathrm{CaO}$ & 4,11 & 8,19 & 6,12 & 3,55 \\
$\mathrm{Na} 2$ & 4,84 & 2,31 & 2,40 & 4,45 \\
$\mathrm{~K}_{2} \mathrm{O}$ & 0,26 & 0,29 & 0,68 & 0,37 \\
$\mathrm{P}_{2} \mathrm{O}_{5}$ & 0,36 & 0,39 & 0,47 & 0,37 \\
$\mathrm{LOI}^{\mathrm{a}}$ & 8,70 & 13,32 & 1,24 & 12,70 \\
\hline Total & 100,01 & 100,01 & 100,00 & 100,00 \\
\hline a Perda por ignição. & & &
\end{tabular}

\subsection{INFLUÊNCIA DO pH}

A remoção dos metais em soluções aquosas é altamente dependente do $\mathrm{pH}$ do meio, pois ele afeta as cargas superficiais das partículas sólidas e o grau de ionização e especiação do adsorvato (Nadaroglu et al., 2010). $\mathrm{O}$ resultado da porcentagem de adsorção de $\mathrm{Cd}(\mathrm{II}) \mathrm{em}$ diferentes valores de $\mathrm{pH}$ está apresentado na Figura 3. Nos experimentos em $\mathrm{pH}$ de 10 e de 12 , os valores de $C_{0}$ e $C_{e}$ ficaram abaixo do limite de detecção do ICP OES, devido à precipitação de $\mathrm{Cd}(\mathrm{II})$, como já indicado na literatura. $^{33,34}$ Essa precipitação foi confirmada usando-se o software CHEAQS (CHemical Equilibria in Aquatic Systems) (Verweij, 2014).
Para o $\mathrm{Cd}(\mathrm{II})$, a maior eficiência de remoção foi em $\mathrm{pH}$ de 7 , em todas as amostras de lama vermelha. Levando-se em conta o desvio padrão associado às medições, pode-se concluir que a eficiência de remoção foi praticamente a mesma para a remoção de $\mathrm{Cd}$ (II) em $\mathrm{pH}$ de 7 tanto para LV quanto para LVT, indicando que a ativação térmica não afeta significativamente a eficiência de remoção de $\mathrm{Cd}(\mathrm{II})$. Contudo, as ativações químicas diminuem muito a capacidade de adsorção da lama vermelha. Embora tenha sido encontrado o valor de $\mathrm{pH}$ ótimo igual a 7, a variação de $\mathrm{pH}$ entre 5,0 e 5,5 usadas nos demais experimentos é um valor amplamente utilizado em estudos de adorção (Nadaroglu et al., 2010; 
Smiljanic et al., 2010; Smiciklas et al., 2014; Santona et al., 2006; Verweij, 2014). Este valor é considerado mais adequado, visto que em $\mathrm{pH}$ ácido não ocorre a precipitação dos metais. Além disso, a realização dos demais experimentos de adsorção com o pH entre 5,0 e 5,5 permite diminuir o consumo de insumos.
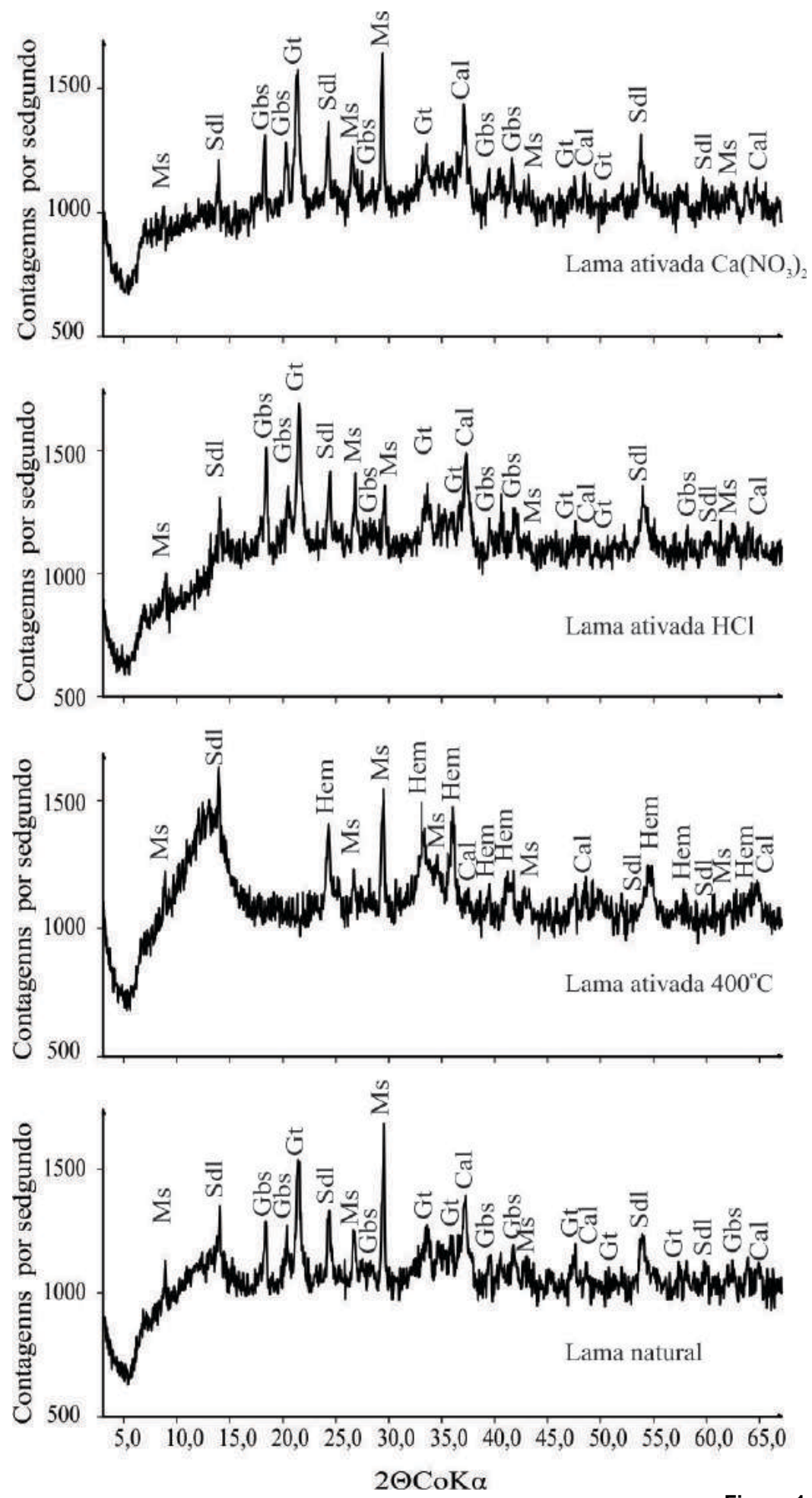

Figura 1

Difratograma e microfotografias dos grãos das diferentes lamas vermalhas utilziadas neste estudo. Cal - calcita, Kln - caulinita, Gbs - gibbsita, Gt - goethita, Hem - hematita, Qtz - quartzo, S - sodalita. 

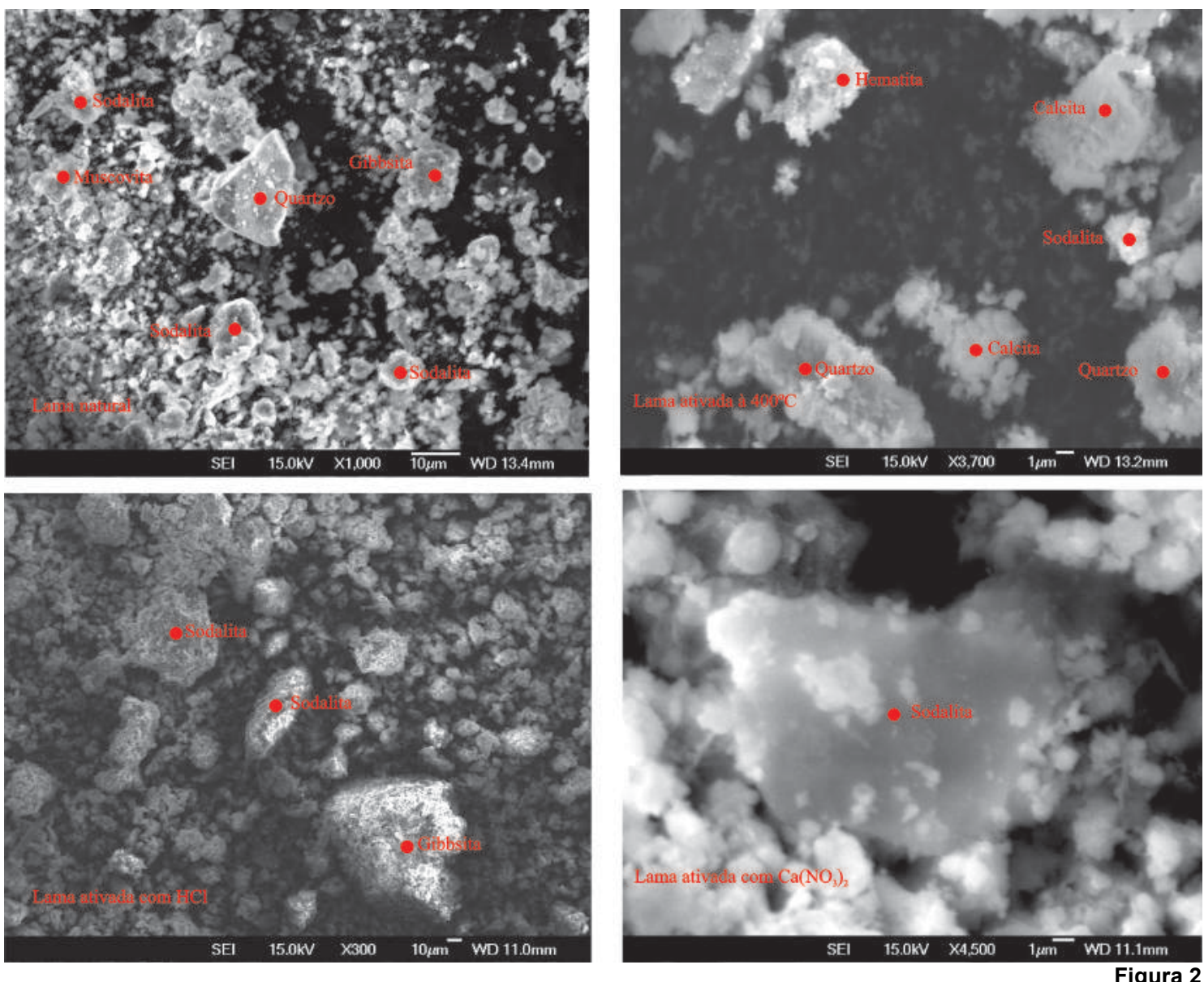

Microfotografias dos grãos das diferentes lamas vermalhas utilizadas neste estudo.

$\unrhd \mathrm{pH} 2 \square \mathrm{pH} 4 \backsim \mathrm{pH} 7$

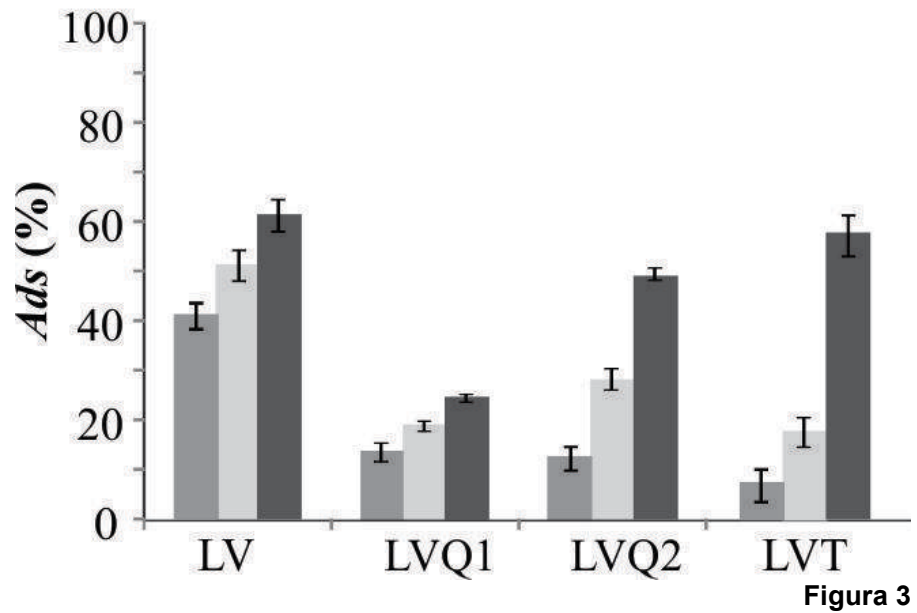

Porcentagem de adsorção de Cd(II) pelas lamas vermelhas em diferente valores de $\mathrm{pH}$ 


\subsection{ISOTERMAS DE ADSORÇÃO}

As isotermas de adsorção descrevem a relação de equilíbrio entre a concentração do adsorvato na fase sólida e sua concentração na fase líquida, em temperatura constante (Souza et al., 2013). O modelo da isoterma de Freundlich (Equação 4) considera a não uniformidade das superfícies reais e descreve a adsorção iônica dentro de certos limites de concentração (Di Bernardo, 2005; Valladares et al., 1998). O modelo da isoterma de Langmuir (Equação 6) assume a existência de um número fixo de sítios acessíveis na superfície do adsorvente, os quais possuem a mesma energia, que independe da presença de espécies adsorvidas nas vizinhanças (Andia, 2009; Tchobanoglous, et al., 2003).

$$
\begin{aligned}
& q_{e}=K_{F} C_{e}^{\frac{1}{n}} \\
& q_{e}=\frac{q_{\operatorname{máx} K_{L} C_{e}}}{1+K_{L} C_{e}}
\end{aligned}
$$

Onde: $\mathrm{K}_{\mathrm{F}}=$ fator de capacidade de adsorção de Freundlich $\left(\left(\mathrm{mmol} \mathrm{g}^{-1}\right) .\left(\mathrm{L} \mathrm{mmol}^{-1}\right) 1 / \mathrm{n}\right) ; 1 / \mathrm{n}=$ parâmetro de intensidade de adsorção de Freundlich; $\mathrm{q}_{\operatorname{máx}}=$ capacidade máxima de adsorção de Lagmuir $\left(\mathrm{mmol} \mathrm{g}^{-1}\right) ; \mathrm{K}_{\mathrm{L}}=$ constante de adsorção no equilíbrio de Lagmuir (mmol 25 $\mathrm{mL}^{-1}$ ).

Os parâmetros das isotermas Freundlich e Langmuir estão apresentados na Tabela $3 . \mathrm{Na}$ Figura 4 são apresentadas as isotermas de adsorção ajustadas pelos modelos de Freundlich e Langmuir. Os melhores ajustes foram obtidos pelo modelo de Freundlich, apresentando valores absolutos de ERM menores que os apresentados pelo modelo de Langmuir. Os mais altos valores de $\mathrm{R}^{2}$ foram obtidos pelo modelo de Freundlich para todas as amostras estudadas, confirmando os valores de ERM (Tabela 3). Assim, o modelo de Freundlich representou mais adequadamente o fenômeno de adsorção de $\mathrm{Cd}(\mathrm{II})$ na LV e suas ativações, para as condições investigadas nesse trabalho.

Tabela 3 - Parâmetros de adsorção obtidos pelos modelos de Freundlich e Langmuir para a adsorção de Cd(II) pela LV e suas

\begin{tabular}{|c|c|c|c|c|c|c|c|c|}
\hline \multirow[b]{2}{*}{ Amostra } & \multicolumn{4}{|c|}{ Freundlich } & \multicolumn{4}{|c|}{ Langmuir } \\
\hline & $\boldsymbol{K}_{\boldsymbol{F}}{ }^{*}$ & $1 / n$ & $R^{2}$ & $E R M$ & $\begin{array}{c}q_{\operatorname{máx}} \\
\left(\mathbf{m m o l ~ g ^ { - 1 }}\right)\end{array}$ & $K_{L} * *$ & $R^{2}$ & $E R M$ \\
\hline $\mathbf{L V}$ & 0,40 & 0,14 & 0,92 & 1,45 & 0,87 & $-0,84$ & 0,90 & 4,28 \\
\hline LVQ1 & 0,17 & 0,28 & 0,95 & 1,68 & 0,14 & $-8,54$ & 0,92 & 8,06 \\
\hline LVQ2 & 0,28 & 0,17 & 0,96 & 1,97 & 0,68 & $-6,41$ & 0,89 & 7,26 \\
\hline LVT & 0,96 & 0,22 & 0,96 & 1,40 & 1,09 & $-3,03$ & 0,90 & 5,47 \\
\hline
\end{tabular}
diferentes ativações, em pH 5,0-5,5.

$*\left[\left(\mathrm{mmol} \mathrm{g}^{-1}\right)\left(25 \mathrm{~mL} \mathrm{mmol}^{-1}\right)^{1 / \mathrm{n}}\right] ; * *\left(25 \mathrm{~mL} \mathrm{mmol}^{-1}\right)$

Os valores de capacidade máxima de adsorção de $\mathrm{Cd}(\mathrm{II})$, em $\mathrm{pH}$ 5,0-5,5, foram de $0,87 \mathrm{mmol} \mathrm{g}^{-1}$ ou $97,44 \mathrm{mg} \mathrm{g}^{-1}$ para LV, 0,14 mmol $\mathrm{g}^{-1}$ ou $15,68 \mathrm{mg} \mathrm{g}^{-1}$ para LVQ1, 0,68 mmol g ${ }^{-1}$ ou $76,16 \mathrm{mg} \mathrm{g}^{-1}$ LVQ2 e $1,04 \mathrm{mmol} \mathrm{g}^{-1}$ ou $107,52 \mathrm{mg} \mathrm{g}^{-1}$ LVT. O valor encontrado para a LVT foi maior que valores encontrados na literatura (Tabela 4) para a adsorção de Cd(II) por lama vermelha tratada com água marinha e $\mathrm{HCl}$, com óxido de ferro e combinada com cimento (Grudić et al., 2013; Ju et al., 2012; Khan et al., 2015;).

Acredita-se que a adsorção na lama vermelha ocorra em grande parte devido à presença da sodalita, um tectossilicato de cálcio e sódio, que possui estrutura porosa e aberta, e é considerado um mineral tipo zeólita, exibindo permanentemente carga negativa em sua superfície (Smiciklas et al., 2014). Esse mineral é o principal responsável pela capacidade de adsorção da lama vermelha, além de outros minerais com importância secundária, tais como óxidos de ferro e de alumínio (Santona et al., 2006). O tratamento químico com $\mathrm{HCl}$ solubiliza uma parte das sodalitas e praticamente todas as micas presentes na lama vermelha (Santona et al., 2006). Assim, sua capacidade de adsorção de $\mathrm{Cd}(\mathrm{II})$ diminui, apesar de haver um aumento na ASE da LVQ1 em relação à LV. Tal fato confirma que a sodalita é o mais importante mineral no processo de adsorção de $\mathrm{Cd}(\mathrm{II})$ pela LV e suas diferentes ativações. 

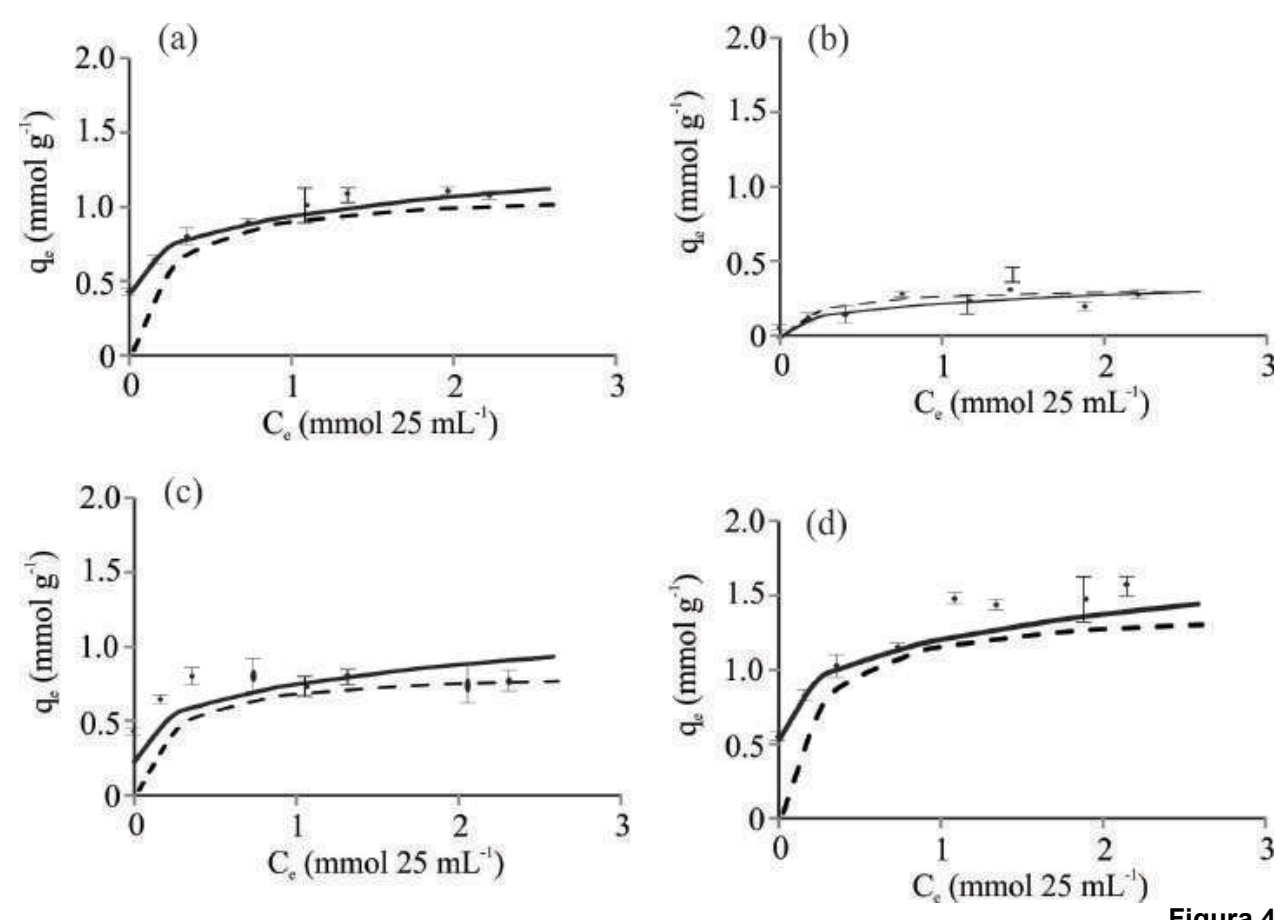

Isotermas de adsorção de Freundlich (linha contínua) e Langmuir (linha trade Cd(II) pela LV(a), LVQ1(b), LVQ2(c) e LVT(d), em pH 5,0-5,5.

Tabela 4 - Comparação da capacidade de adsorção de $\mathrm{Cd}(\mathrm{II})\left(\mathrm{mg} \mathrm{g}^{-1}\right)$ para as diferentes lamas vermelhas com outros estudos.

\begin{tabular}{c|c|c|c}
\hline & Tratamento & Capacidade de adsorção & Referência \\
\hline \multirow{4}{*}{ Cd(II) } & Natural & 97,44 & Presente estudo \\
& Ácido clorídrico & 15,68 & \\
\cline { 2 - 4 } & Nitrato de cálcio & 76,16 & GRUDIĆ et al.2013 \\
& Térmico a $400^{\circ} \mathrm{C}$ & 107,52 & JU et al. 2012 \\
& Água marinha e HCl & 24,64 & KHAN et al. 2015 \\
\hline
\end{tabular}

\subsection{CINÉTICA DA REAÇÃO DE ADSORÇÃO}

O tempo de equilíbrio do processo de adsorção de $\mathrm{Cd}(\mathrm{II})$ pela $\mathrm{LV}$ e suas diferentes ativações, em $\mathrm{pH}$ 5,0-5,5, é apresentado na Figura 5. Pode-se observar que a estabilização iniciou-se a partir de 420 minutos. Os modelos de pseudo-primeira ordem e pseudo-segunda ordem foram aplicados para explicar a cinética da reação e analisar o mecanismo de controle do processo de adsorção. A equação de pseudoprimeira ordem de Lagergren está apresentada na Equação 6 (Ho \& Mckay, 1998). A equação para o modelo cinético de pseudo-segunda ordem é representada pela Equação 7.

$$
\begin{aligned}
& \frac{d q_{t}}{d t}=k_{1}\left(q_{e}-q_{t}\right) \\
& \frac{d q_{t}}{d t}=k_{2}\left(q_{e}-q_{t}\right)^{2}
\end{aligned}
$$

Onde: $\mathrm{q}_{\mathrm{e}}=$ quantidade adsorvida no equilíbrio $\left(\mathrm{mmol} \mathrm{g}^{-1}\right) ; \mathrm{q}_{\mathrm{t}}=$ quantidade adsorvida no tempo $\mathrm{t}$ $(\mathrm{mmol} \mathrm{g}) ; \mathrm{k}_{1}=$ constante de velocidade de pseudo-primeira ordem $\left(\mathrm{min}^{-1}\right) ; \mathrm{k}_{2}$ : constante de velocidade de pseudo segunda-ordem de adsorção (g (mmol.min $\left.)^{-1}\right)$. 


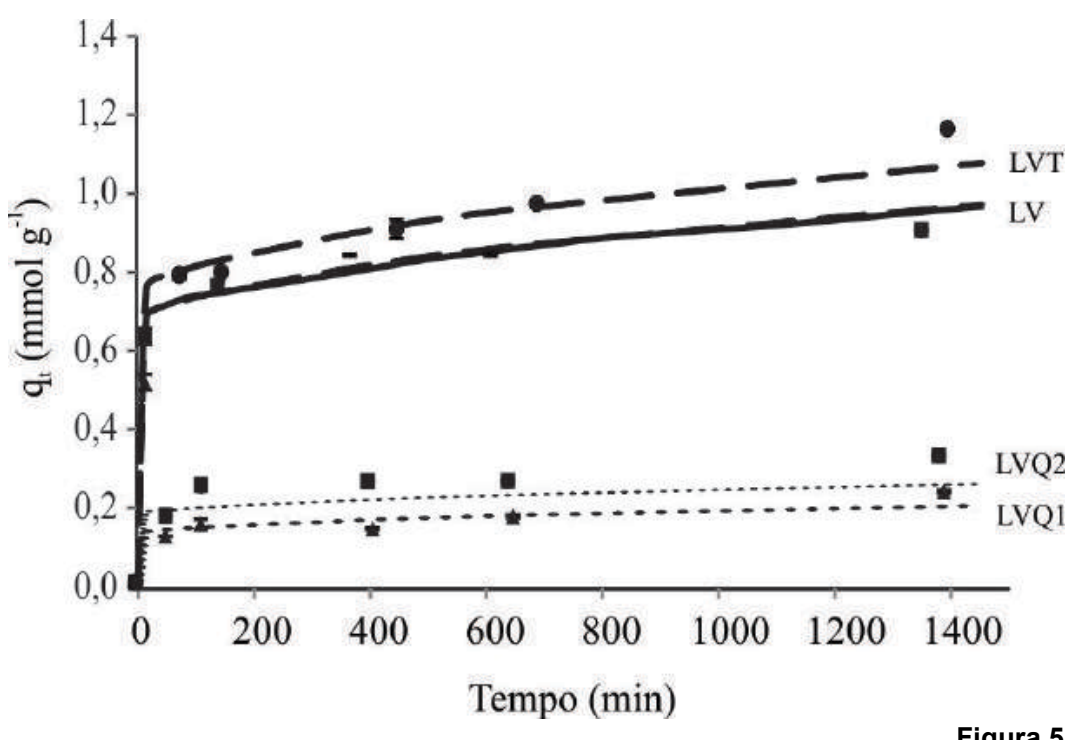

Tempo de equilíbrio da adsorção de Cd(II) pela LV(a), LVQ1(a), LVQ2(c) e LVT(d), em pH 5,0-5,5.

A partir do ajuste dos modelos cinéticos aos dados experimentais da adsorção, em pH 5,0-5,5, foram obtidos os parâmetros apresentados na Tabela 5. Comparando os parâmetros dos modelos pseudo-primeira ordem e pseudosegunda ordem, e sua adequação aos dados experimentais com base nos valores de ERM e os valores de $\mathrm{R}^{2}$, o modelo de pseudo-segunda ordem é o que se ajusta melhor aos dados experimentais. O melhor ajuste ao modelo de pseudo-segunda ordem sugere processos de adsorção química, envolvendo processos de doação ou troca de elétrons entre o adsorvato e o adsorvente, como forças covalentes e de troca iônica (Alves, 2012).

Tabela 5 - Parâmetro cinéticos obtidos pelos modelos de pseudo-primeira ordem e pseudo-segunda ordem para a adsorção de $\mathrm{Cd}$ (II) pela LV e suas diferentes ativações, em pH 5,0-5,5.

\begin{tabular}{|c|c|c|c|c|}
\hline Amostra & $\mathbf{L V}$ & LVQ1 & LVQ2 & LVT \\
\hline$q_{e(e x p)}\left(\mathrm{mmol} \mathrm{g}^{-1}\right)$ & 0,93 & 0,36 & 0,32 & 0,97 \\
\hline \multicolumn{5}{|c|}{ Pseudo-primeira ordem } \\
\hline$q_{e}\left(\mathrm{mmol} \mathrm{g}^{-1}\right)$ & 0,10 & 0,07 & 0,13 & 0,12 \\
\hline$k_{l} \times 10^{3}\left(\min ^{-1}\right)$ & $-0,92$ & $-1,61$ & 7,14 & $-2,53$ \\
\hline$R^{2}$ & 0,48 & 0,15 & 0,98 & 0,97 \\
\hline$E R M$ & 107,97 & 71,73 & 69,22 & 189,34 \\
\hline \multicolumn{5}{|c|}{ Pseudo-segunda ordem } \\
\hline$q_{e}\left(\mathrm{mmol} \mathrm{g}^{-1}\right)$ & 0,92 & 0,35 & 0,26 & 0,97 \\
\hline$k_{2} \times 10^{3}\left(\mathrm{~g}(\mathrm{mmol} \mathrm{min})^{-1}\right)$ & 0,14 & 0,04 & $-143,23$ & 0,09 \\
\hline$R^{2}$ & 0,99 & 0,94 & 0,99 & 0,99 \\
\hline$E R M$ & 4,07 & 14,74 & 22,2 & 5,93 \\
\hline
\end{tabular}

\subsection{TERMODINÂMICA}

O estudo da termodinâmica permite verificar a natureza das reações de adsorção, endotérmicas ou exotérmicas. A variação da energia livre padrão ou energia de Gibbs $\left(\Delta G^{\circ}\right)$ em J.mol ${ }^{-1}$ foi calculada a partir da Equação 8, para a condição de equilíbrio termodinâmico (Önal, 2006).

$$
\Delta G^{0}=-R T \ln K_{C}
$$

Onde: $\mathrm{R}=$ constante do gás $\left(\mathrm{J}(\mathrm{mol} . \mathrm{K})^{-1}\right) ; \mathrm{kc}=$ constante de equilíbrio; $\mathrm{T}=$ temperatura em $\mathrm{K}$.

$\mathrm{O}$ valor de $\mathrm{k}_{\mathrm{c}}$ foi obtido por meio da Equação 9.

$$
k_{c}=\frac{C_{A e}}{C_{e}}
$$

Onde: $\mathrm{C}_{\mathrm{Ae}}$ e $\mathrm{C}_{\mathrm{e}}=$ concentração de equilíbrio de íons do adsorvato no adsorvente, e na solução $\left(\mathrm{mmol} \mathrm{mL} \mathrm{m}^{-1}\right)$, respectivamente. 
A variação de entalpia $\left(\Delta \mathrm{H}^{\circ}\right)$ em $\mathrm{kJ} \mathrm{mol}^{-1}$ e a variação de entropia $\left(\Delta S^{o}\right)$ em $\mathrm{J}(\text { mol.K })^{-1}$ de adsorção foram obtidas a partir de equação van't Hoff (Equação 10) (ÖNAL, 2006). As constantes foram obtidas a partir da inclinação e intercepção da reta no gráfico de $\ln \mathrm{K}_{\mathrm{c}}$ em função de $1 / T$.

$$
\ln k_{c}=-\frac{\Delta H_{a d s}^{0}}{R T}+\frac{\Delta S^{0}}{R}
$$

Onde: $-\Delta H^{\circ} / \mathrm{R}=$ coeficiente angular da reta no gráfico de van't Hoff; $\Delta \mathrm{S} / \mathrm{R}=$ intercepto do gráfico de van't Hoff.

A porcentagem de adsorção de $\mathrm{Cd}(\mathrm{II})$ pela $\mathrm{LV}$ e suas ativações em diferentes temperaturas $(303 \mathrm{~K}, 313 \mathrm{~K}$ e $323 \mathrm{~K})$, em pH 5,0-5,5, está apresentada na Figura 6. É possível perceber que a porcentagem de adsorção de $\mathrm{Cd}$ (II) aumenta com a elevação da temperatura. Os parâmetros termodinâmicos obtidos estão apresentados na Tabela 6. Os valores positivos para $\Delta \mathrm{H}^{\circ}$ indicam ocorrência de reações endotérmicas, e valores positivos de $\Delta \mathrm{S}^{\circ}$ indicam afinidade das lamas vermelhas por Cd(II) (Chowdhury et al., 2011). Para $\Delta \mathrm{G}^{\circ}$ observam-se valores negativos $\mathrm{e}$ positivos, os negativos confirmam a viabilidade do processo e natureza espontânea de adsorção, que é o caso a $323 \mathrm{~K}$ para LV, e a $313 \mathrm{~K}$ e $323 \mathrm{~K}$ para LVT. O crescimento do valor absoluto de $\Delta \mathrm{G}^{\circ}$ com o aumento da temperatura sugere aumento da afinidade de adsorção de $\mathrm{Cd}(\mathrm{II})$, pela LVT, com a temperatura (Chowdhury et al., 2011).

Tabela 6 - Parâmetros termodinâmicos da adsorção de Cd(II) pela LV e suas diferentes ativações, em pH 5,0-5,5.

\begin{tabular}{|c|c|c|c|c|c|}
\hline \multirow{2}{*}{ Amostra } & \multirow{2}{*}{$\begin{array}{c}\Delta \mathrm{H}^{\circ} \\
\left(\mathrm{kJ} \mathrm{mol}^{-1}\right)\end{array}$} & \multirow{2}{*}{$\begin{array}{c}\Delta \mathrm{S}^{\circ} \\
\left(\mathrm{J}(\mathrm{mol} \mathrm{K})^{-1}\right)\end{array}$} & \multicolumn{3}{|c|}{$\Delta G^{\circ}\left(\mathrm{kJ} \mathrm{mol}^{-1}\right)$} \\
\hline & & & $303 \mathrm{~K}$ & $313 \mathrm{~K}$ & $323 \mathrm{~K}$ \\
\hline $\mathbf{L V}$ & 9,34 & 29,71 & 0,30 & 0,14 & $-0,30$ \\
\hline LVQ1 & 42,48 & 119,35 & 6,52 & 4,70 & 4,16 \\
\hline LVQ2 & 10,21 & 13,93 & 6,06 & 5,71 & 5,79 \\
\hline LVT & 13,49 & 43,89 & 0,16 & $-0,16$ & $-0,72$ \\
\hline
\end{tabular}

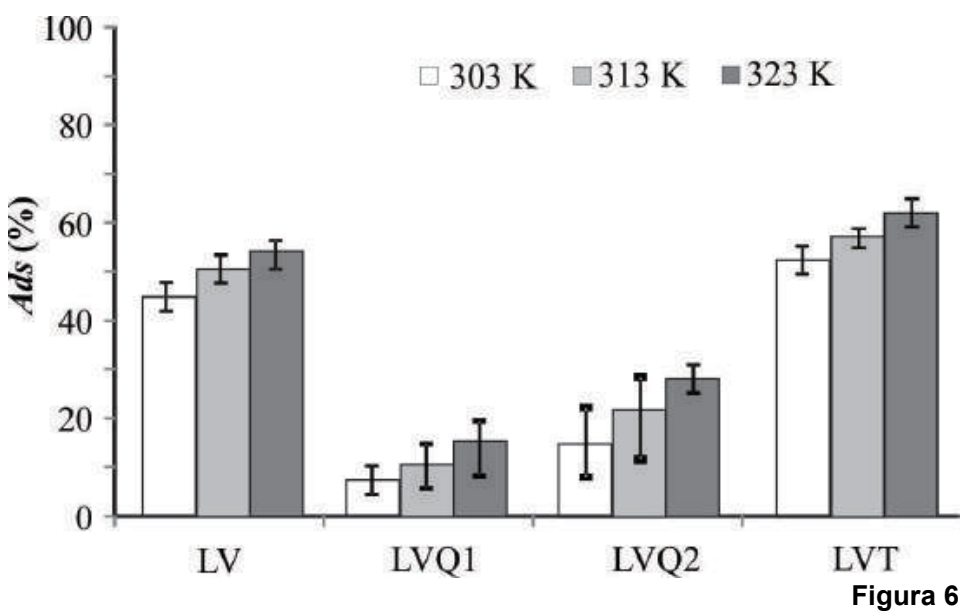

Porcentagem de adsorção de Cd(II) em diferentes temperaturas pela LV(a), LVQ1(b), LVQ2(c) e LVT(d), em pH 5,0-5,5.

\section{CONCLUSÕES}

Neste estudo, a caracterização da lama vermelha e adsorção de $\mathrm{Cd}(\mathrm{II})$ pela lama vermelha natural (LV) e em diferentes ativações químicas ( $\mathrm{HCl} \mathrm{0,05} \mathrm{mol} \mathrm{L}^{-1}$ - LVQ1 e $\mathrm{Ca}\left(\mathrm{NO}_{3}\right)_{2}$ $\left.0,1 \mathrm{~mol} \mathrm{~L}^{-1}-\mathrm{LVQ} 2\right)$ e térmica $\left(400^{\circ} \mathrm{C}\right)$ foram avaliadas. $\mathrm{O}$ tratamento térmico da $\mathrm{LV}$ a $400^{\circ} \mathrm{C}$ aumentou a área superficial específica devido à mudança de goethita para hematita e gibbsita para alumina e decréscimo dos poros das partículas. A mais alta porcentagem de remoção de Cd(II) foi observada na LVT em valores de $\mathrm{pH}$ 5,0-5,5. A remoção de $\mathrm{Cd}(\mathrm{II})$ em soluções aquosas obedece ao modelo de Freundlich, com máxima capacidade de adsorção relacionada à LVT $\left(1,04 \mathrm{mmol} \mathrm{g}^{-1}\right.$ ou $\left.107,52 \mathrm{mg} \mathrm{g}^{-1}\right)$. O tempo de equilíbrio do processo de adsorção de $\mathrm{Cd}(\mathrm{II})$ 
pela LV e suas diferentes ativações, em $\mathrm{pH}$ 5,05,5 , iniciou-se a partir de 420 minutos, sendo que o modelo de pseudo-segunda ordem se ajusta melhor aos dados experimentais, sugerindo processos de adsorção química. A porcentagem de adsorção de Cd(II) aumenta com $\mathrm{o}$ incremento de temperatura e os valores de $\Delta \mathrm{H}^{\circ}$ e $\Delta \mathrm{S}^{\circ}$ positivos indicam que a reação de

\section{AGRADECIMENTOS}

Os autores agradecem a Fundação de Amparo à Pesquisa do Estado de São Paulo (FAPESP Processos 2009/02374-0 e 2013/00994-6), Conselho Nacional de Desenvolvimento

\section{REFERÊNCIAS BIBLIOGRÁFICAS}

Ahmaruzzaman, M. 2011. Industrial wastes as lowcost potential adsorbents for the treatment of wastewater laden with heavy metals. Adv. Colloid Interface Sci., 166: 36-59.

Akin, I.; Arslan, G.; Tor, A.; Ersoz, M.; Cengeloglu, Y. 2012. Arsenic (V) removal from underground water by magnetic nanoparticles synthesized from waste red mud. J. Hazard. Mater., 235-236: 6268.

Alves, C. C. O. 2012. Remoção de aminoácidos aromáticos de soluções aquosas por adsorvente preparado de resíduo agrícola. Tese de Doutorado, Universidade Federal de Minas Gerais, Faculdade de Farmácia, Belo Horizonte, Brasil, 209f.

Andia, P. M. 2009. Remoção de boro de águas e efluentes de petróleo por adsorção. Tese de Doutorado, Pontifícia Universidade Católica do Rio de Janeiro, Brasil, 138f.

Antunes, M. L. P; Couperthwaite, S. J; Conceição, F. T; Jesus, C. P. C; Kiyohara, P. K; Coelho, A. C. V; Frost, R. L. 2012. Red Mud from Brazil: Thermal Behavior and Physical Properties. Industrial \& Engineering Chemistry Research, 51: 775-779.

Apak, R.; Guclu, K.; Turgut, M. H. 1998a. Modeling of Copper (II), Cadmium (II), and Lead (II) adsorption on red mud. J. Colloid Interface Sci., 23: $122-130$.

Apak, R.; Tütem, E.; Hügül, M.; Hizal, J. 1998b. Heavy metal cation retention by unconventional sorbents (red muds and fly ashes). Water Res., 32: 430-440.

Behnamfard, A.; Salarirad, M. M.; Vegliò, F. 2014. Removal of $\mathrm{Zn}$ (II) ions from aqueous solution by ethyl xanthate impregnated activated carbons. Hydrometallurgy., 144-145: 39-53.

Bhattacharya, K. G.; Gupta, S. S. 2008. Influence of acid activation on adsorption of $\mathrm{Ni}$ (II) and $\mathrm{Cu}(\mathrm{II})$ adsorção é endotérmica e afinidade entre o $\mathrm{Cd}(\mathrm{II})$ e a LV e suas diferentes ativações. Assim, este estudo sugere que o uso da LV ou com diferentes ativações, especialmente a ativada termicamente, tem um potencial de uso no tratamento de efluentes ou remediação de solos contaminados de áreas com atividades industriais.

Científico e Tecnológico $(\mathrm{CNPq}$ - Processo 480555/2009-5) e a Companhia Brasileira de Alumínio (CBA).

on kaolinite and montmorillonite: Kinetic and thermodynamic study. Chem. Eng. J., 136: 1-13.

Brunauer, S.; Emmett, P. H.; Teller, E. 1938. Adsorption of Gases in Multimolecular Layers. $J$. Am. Chem. Soc., 60: 309-319.

Cengeloglu, Y.; Tor, A.; Ersoz, M.; Turgut, M. H.; Sep. Purif. Technol., v.51, 2006, p.374.

Chowdhury, S.; Mishra, R.; Saha, P.; Kushwaha, P. 2011. Adsorption thermodynamics, kinetics and isosteric heat of adsorption of malachite green onto chemically modified rice husk. Desalination, 265: 159-168

Conceição, F. T.; Pichinelli, B. C.; Silva, M. S. G.; Moruzzi, R. B. ; Menegario, A. A. ; Antunes, M. L. P.2016. $\mathrm{Cu}(\mathrm{II})$ adsorption from aqueous solution using red mud activated by chemical and thermal treatment. Environ. Earth Sci., 75: 1-7.

Costa, E. T. S; Guilherme, L. R. G; Curi, N; Lopes, G; Visoli, E. L; Oliveira, L. C. A. 2009. Caracterização de subproduto da indústria de alumínio e seu uso na retenção de cádmio e chumbo em sistemas monoelementares. Quim. Nova. 32: 868-847.

Di Bernardo, L. 2005. Métodos e técnicas de tratamento de água, São Carlos: RiMA. $788 f$.

Embrapa. 1997. Manual de Métodos de Análise de Solo, 2nd ed. 212f.

Erdem, M.; Altundogan, H. S.; Tumen, F. 2004. Removal of hexavalent chromium by using heatactivated bauxite. Min. Eng., 17: 1045-1052.

Fu, J.; Song, R.; Mao,W.; Wang, Q.; An, S.; Zeng, Q.; Zhua, H. 2010. Adsorption of disperse blue 2BLN by microwave activated red mud. Environ. Prog. Sustainable Energy. 30: 558-566.

Geyikçi, F.; Kiliç, E.; Çoruh, S.; Elevli, S. 2012. Modeling of lead adsorption from industrial sludge leachate on red mud by using RSM and ANN. Chem. Eng. J. 183: 53-59. 
Grudić, V. V.; Brašanac, S.; Vukašinović-Pešić, V. L.; Blagojević, N. Z. 2013. Sorption of cadmium from water using neutralized red mud and activated neutralized red mud. ARPN J. Eng. Appl. Sci., 8: 933-943.

Gupta, V.K.; Sharma, S. 2002. Removal of cadmium and zinc from aqueous solutions using red mud. Environ. Sci. Technol., 36: 3612-3617.

Hind, A. R.; Bhargava, S. K.; Grocott, S. C. 1999. The surface chemistry of Bayer process solids: a review. Colloids Surf. A., 146: 359-374.

Ho, Y. S. Mckay, G.; 1998. A comparison of chemisorption kinetic models applied to pollutant removal on various sorbents. Trans. IChemE., 76:332.

Jesus, C. P. C. 2011. Caracterização da lama vermelha e sua aplicabilidade na adsorção do corante têxtil reativo azul 19. Dissertação de Mestrado, Universidade Estadual Paulista, Bauru. $131 \mathrm{f}$.

Ju, S.; Lu, S.; Peng, J.; Zhang, L.; Srinivasakannan, C.; Guo, S.; Li, W. 2012. Removal of cadmium from aqueous solutions using red mud granulated with cement. Trans. Nonferrous Met. Soc. China, 22: 3140-3146.

Khan, T. A.; Chaudhry, S. A.; Ali, Imran. 2015. Equilibrium uptake, isotherm and kinetic studies of $\mathrm{Cd}(\mathrm{II})$ adsorption onto iron oxide activated red mud from aqueous solution. J. Mol. Liq. 202: 165175.

Liang, W.; Couperthwaite, S. J.; Kaur, G.; Yan, C.; Jonhstone, D. W.; Millar, G. J. 2014. Effect of strong acids on red mud structural and fluoride adsorption properties. J. Colloid Interface Sci., 423: 158-165.

Nadaroglu, H.; Kalkan, E.; Demir, N. 2010. Removal of copper from aqueous solution using red mud. Desalination. 251: 90-95.

Önal, Y.; J. 2006. Kinetics of adsorption of dyes from aqueous solution using activated carbon prepared from waste apricot. Hazard. Mater.B., 137: 17191728.

Palmer, S. J.; Nothling, M.; Bakon, K. H.; Frost, R.L. 2010. Thermally activated seawater neutralised red mud used for removal of arsenate, vanadate and molybdate from aqueous solutions. J. Colloid Interface Sci., 342: 147-154.

Pichinelli, B. C.; Silva, M. S. G.; Conceição, F. T.; Menegario, A. A.; Antunes, M. L. P.; Navarro, G. R. B.; Moruzzi, R. B. 2017. Adsorption of Ni(II), $\mathrm{Pb}$ (II) and $\mathrm{Zn}(\mathrm{II})$ on $\mathrm{Ca}\left(\mathrm{NO}_{3}\right)_{2}$-neutralised red mud. Water, Air, Soil Pollut., 228: 1-13.

Pulford, I. D.; Hargreaves, J. S. J.; Durisová, J.; Kramulova, B.; Girard, C.; Balakrishnan, M.; Batra, V. S.; Rico, J. L. 2012. Carbonised red mud - A new water treatment product made from a waste material. J. Environ. Manage., 100: 59-64.

Rai, S.; Wasewar, K. L.; Mukhopadhyay, J.; Yoo, C. K.; Uslu, H. 2012. Neutralization and utilization of red mud for its better waste management. Archives of Environ. Sci., 6: 13-33.

Ratnamala, G. M.; Vidya Shetty, K.; Srinikethan, G. 2012. Removal of remazol brilliant blue dye from dye-contaminated water by adsorption using red mud: equilibrium, kinetic and thermodynamic studies. Water, Air, Soil Pollut., 223: 6187-6199.

Sahu, R. C.; Patel, R.; Ray, B. C. 2011. Adsorption of $\mathrm{Zn}$ (II) on activated red mud: Neutralized by $\mathrm{CO}_{2}$. Desalination, 266: 93-97.

Santona, L.; Castaldi, P.; Melis, P. 2006. Evaluation of the interaction mechanisms between red mud and heavy metals. J. Hazard. Mater., 136: 324329.

Silva Filho, E. B.; Alves, M. C. M.; Motta, M. 2007. Lama vermelha da indústria de beneficiamento de alumina: produção, características, disposição e aplicações alternativas. Rev. Mater., 12: 322-338.

Smiciklas, I.; Smiljanic, S.; Peric-Grujic, A.; M. Šljivic-Ivanovic, M.; Mitric, M.; Antonovic, D. 2014. Effect of acid treatment on red mud properties with implications on $\mathrm{Ni}$ (II) sorption and stability. Chem. Eng. J, 242: 27-35.

Smiljanic, S.; Smiciklas, I.; Peric-Grujic, A.; Loncar, B.; Mitri, M. 2010. Rinsed and thermally treated red mud sorbents for aqueous $\mathrm{Ni}^{2+}$ íons. Chem. Eng. J., 162: 75-83.

Souza, K. C.; Antunes, M. L. P.; Conceição, F. T. 2013. Adsorção do corante Reativo Azul 19 em solução aquosa por Lama Vermelha tratada quimicamente com peróxido de hidrogênio. Quim. Nova, 36: 651-656.

Souza, K. C.; Antunes, M. L. P.; Couperthwaite, S. J.; Conceição, F. T.; Barrs, T. R.; Frost, R. 2013. Adsorption of reactive dye on seawaterneutralised bauxite refinery residue. J. Colloid Interface Sci., 396: 210-214.

Tchobanoglous, G.; Burton, F. L.; Stensel, H. D. 2003. Wastewater engineering: Treatment and reuse. Metcalf \& Eddy, Inc., 4th ed., Boston: McGraw-Hill, 1848f.

Valladares, G. S.; Pereira, M. G.; Alves, G. C. 1998. Aplicação de duas isotermas de adsorção de boro em solos de baixada do estado do Rio de Janeiro. Rev. Bras. Cienc. Solo, 22: 361-365.

Verweij, W. 2014. CHEAQS PRO; A program for calculating CHemical Equilibria in AQuatic Systems; Netherlands, 1999-2014.

Zhou, Y.; Haynes, R. J. 2011. A Comparison of Inorganic Solid Wastes as Adsorbents of Heavy Metal Cations in Aqueous Solution and Their Capacity for Desorption and Regeneration. Water, Air, Soil Pollut., 218: 457-470. 